\title{
Overview of electron polarimetry
}

\section{Charles K. Sinclair}

Cornell University

Ithaca, New York, USA 14853

E-mail: cks260cornel1.edu

The 1956 discovery of parity non-conservation in weak interactions led directly and rather quickly to the development of electron polarimeters based on both Mott and M $\phi 1$ ler scattering. These early polarimeters appeared well before the first polarized beams were generated, almost two decades later. Compton scattering was initially used to generate polarized gamma beams in the late 1960s, before the technique was adapted to measuring the polarization of stored beams in storage rings in the 1970s. These three methods of measuring the polarization of electron beams are now all in wide general use, and have evolved considerably since the early polarimeter designs. Parity violation has now been developed into a tool for studying the detailed structure of nucleons, which has led directly to very demanding requirements for high precision polarimeters. We will briefly describe some of the recent developments in these polarimeters, mention their limitations, and briefly indicate where the field may move in the near future.

XVth International Workshop on Polarized Sources, Targets, and Polarimetry September 9-13, 2013

Charlottesville, Virginia, USA 


\section{Introduction}

This brief overview is restricted to electron polarization measurement techniques used in the nuclear and particle physics fields. There are ways to measure electron polarization involving interactions with atoms or surfaces, used outside the nuclear and particle fields, and possibly for polarized source development, which are inapplicable to the present discussion. Scientific communication between the eastern and western nations was not all that good during the early developments in electron polarimetry. This overview covers developments made principally in the US and western Europe.

Electrons experience only the electromagnetic, weak, and gravitational forces. As the weak and gravitational forces are unlikely to provide good polarization measurement tools, we have only the electromagnetic interactions available to us. This gives us three basic schemes - scattering of electrons by other electrons - M $\phi 1 l e r$ scattering [1], scattering of electrons by photons Compton scattering [2], or scattering of electrons by nuclei - Mott scattering [3]. Each of these three basic scattering mechanisms was understood relatively early, including the significant polarization asymmetries associated with each process. Being electromagnetic processes, their analyzing power is calculable with great precision. As a consequence the uncertainties in measuring electron polarization arise from backgrounds, systematic effects, various "dirt" effects, and possibly statistics.

The underlying science that initially drove the need for, and currently drives the required precision of electron polarimetry is parity violation. The reasons for this are fairly straightforward. In the years before parity violation in the weak interactions was proposed and confirmed, Mott scattering was the only demonstrated way to polarize electrons or measure their polarization. This reality greatly restricted the possibilities for doing physics with polarized electrons. With the arrival of parity violation, electron polarization measurement became a widely used tool to understand the weak interactions. When polarized electron sources for accelerators appeared in 1974, experiments not involving parity violation required either a polarized target or measurement of an angular distribution, and the uncertainly in knowledge of the polarization was only one contribution to the experimental uncertainty. In contrast, uncertainty in knowledge of the beam polarization is generally a major contributor to the overall experimental uncertainty in parity violation measurements. The growing use of parity violation to study nuclear and nucleon structure is the present driving force for improvements in electron polarimetry.

\section{The state of electron polarization measurements in late 1956}

Before looking at the early measurements of electron (and positron) polarization in beta decay, it is worth reviewing what was known about electron polarization and its measurement at the time parity non-conservation was first suggested, in late 1956. After a number of negative or inconclusive experiments, the Mott scattering asymmetry was clearly demonstrated in a double scattering measurement published in early 1943 [4]. This work was the Ph.D, thesis of Clifford Shull, who went on to share the Nobel Prize in Physics in 1994. Shull measured the Mott asymmetry in $90^{\circ}$ scattering of a $400 \mathrm{keV}$ beam from a gold foil. At the time of this work, the Mott asymmetry had been calculated only at $90^{\circ}$ over a limited energy range. Shull's apparatus is shown in figure 1, and the result of his measurement is compared to the then extant calculations of the asymmetry in figure 2. It is fascinating to realize that Shull's apparatus was constructed many years before components like knife-edge vacuum flanges and ceramic-tometal feedthroughs were commercially available. The "quadrant blinkers" referred to in the 
figure were used to center the beam at the entrance to the apparatus. Each of the four electrically isolated quadrants was returned to ground through a capacitor and a neon blub in parallel. When beam interception charged the capacitor to the breakdown voltage of the neon bulb, the bulb flashed. Steering was accomplished by equalizing the blinking rate from the four quadrants! The difference in the calculated asymmetries shown in figure 2 illustrates the difficulty of the calculation prior to the availability of digital computers. The first good calculations of this asymmetry were made by Sherman in 1955, utilizing a UNIVAC, and the Mott asymmetry has since been known as the "Sherman function" [5].

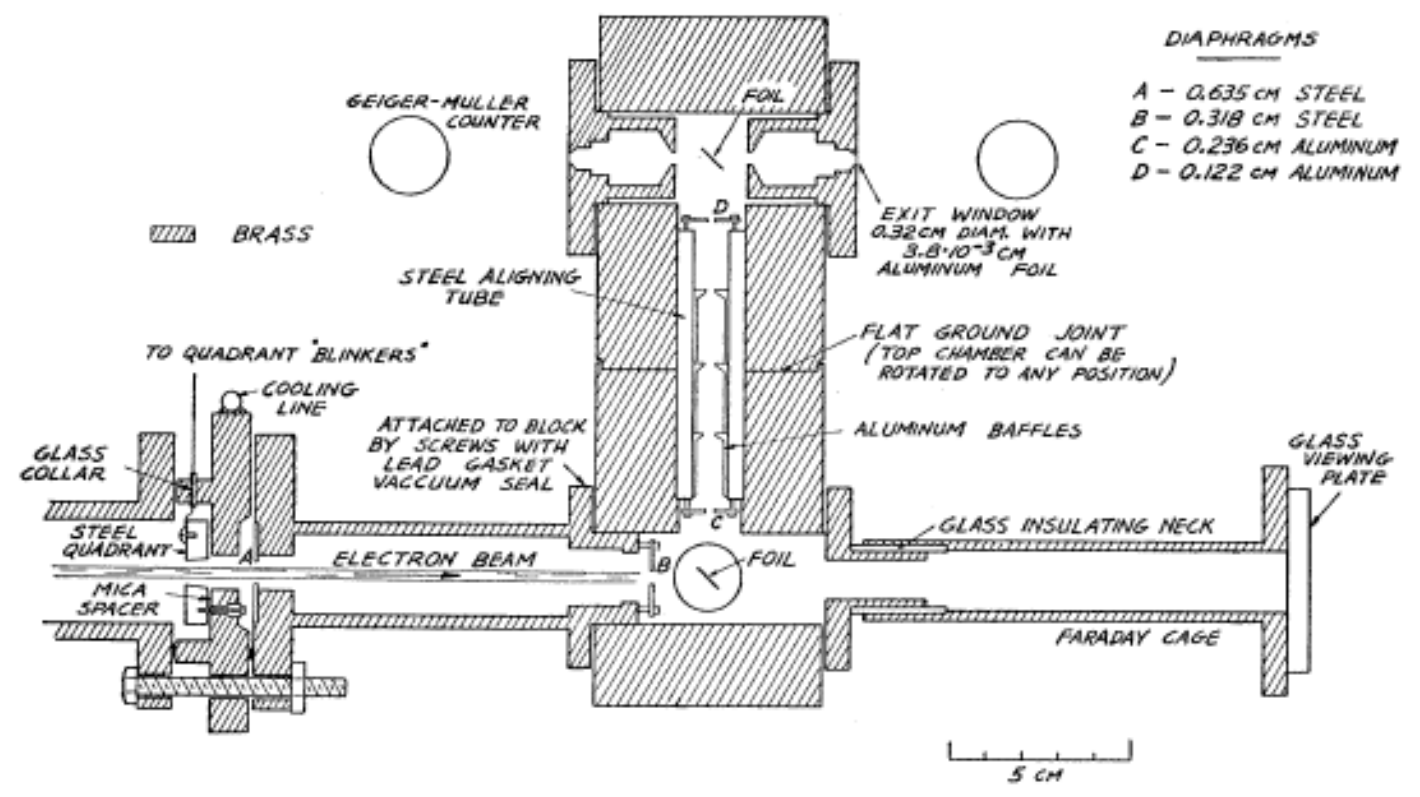

Figure 1. The double scattering apparatus used by Shull et al. to demonstrate the Mott scattering asymmetry of $400 \mathrm{keV}$ electrons

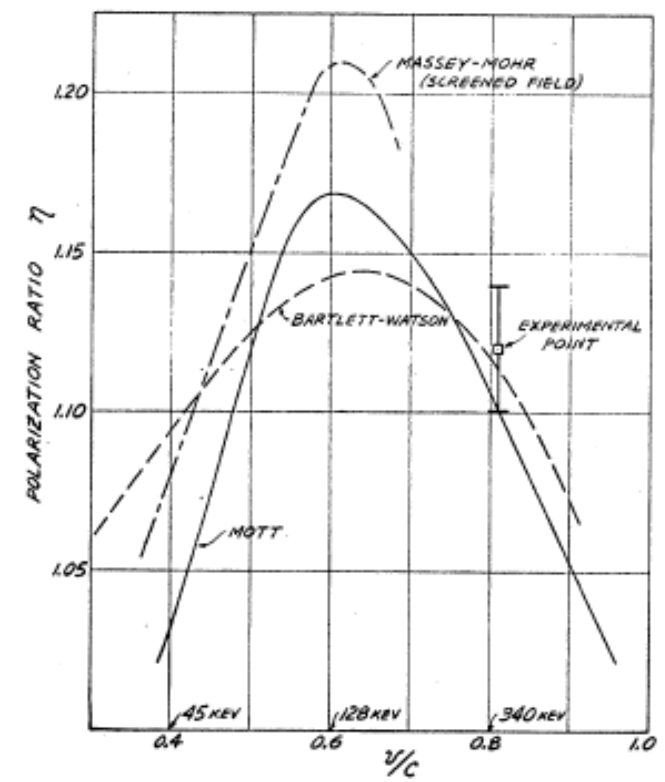

Figure 2. Shull's et al.'s measurement of the $400 \mathrm{keV}$ Mott asymmetry at $90^{\circ}$, with the theoretical calculations of the time 
Finally, as an example of an experiment that used Mott scattering as a polarized electron source as well as a polarization analyzer, we note the first measurement of the magnetic moment of the free electron by Louisell, Pidd, and Crane [6]. In the early 1950s the anomalous magnetic moment of the electron bound in atoms was known about, but it was not clear that the value would be the same for free electrons. The apparatus of Louisell et al. (his Ph.D. thesis) is shown in figure 3. They measured the spin precession of electrons initially polarized by Mott scattering in the magnetic field of a long solenoid, obtaining a value of $2.00+/-0.01$ for the $g$ value. This experiment demonstrated the principle, and it was later improved to provide an accurate measurement of the anomaly.

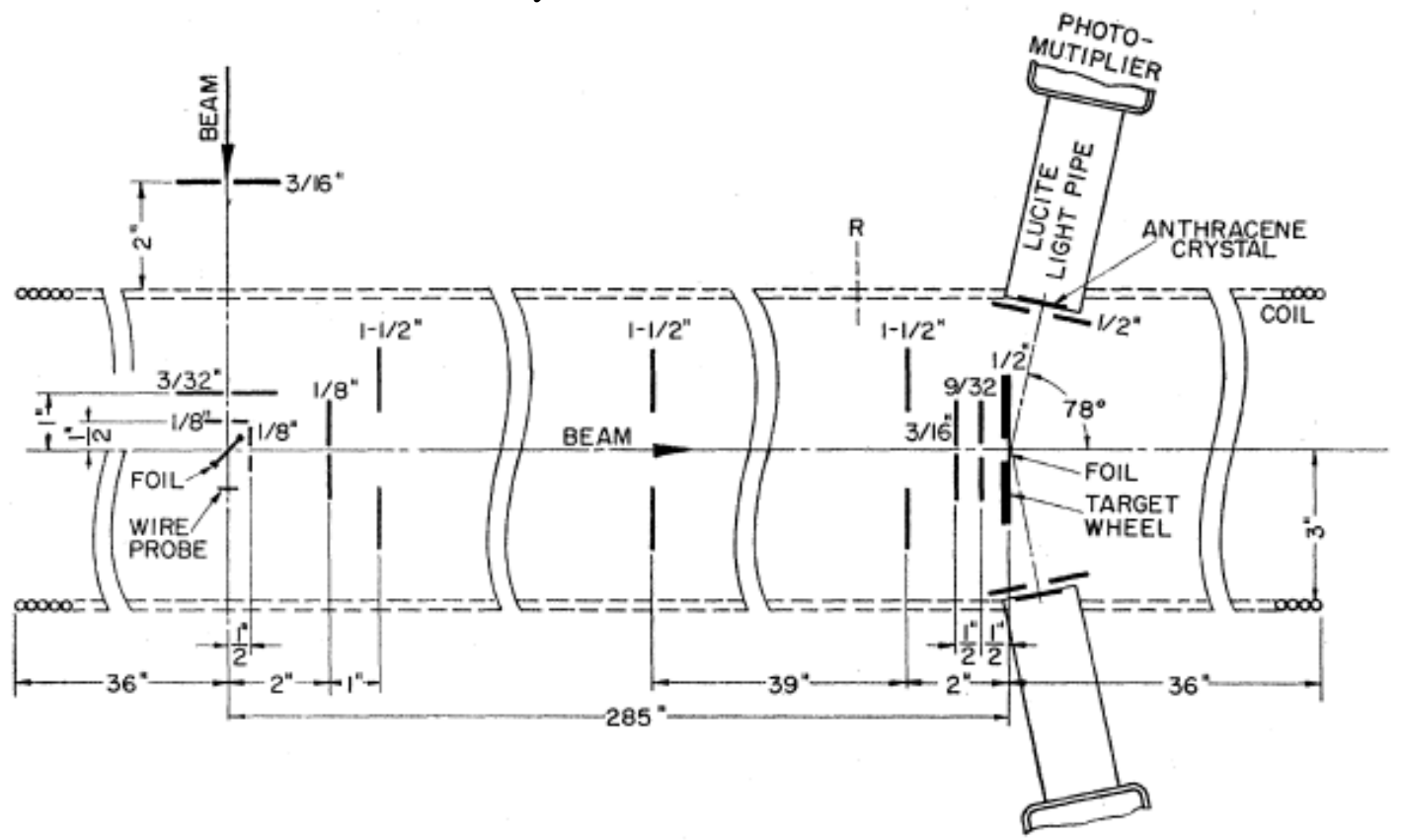

Figure 3. The apparatus of Louisell et al. for the measurement of the free electron g value.

\section{The first measurements of parity violation in the weak interactions}

The famous Lee and Yang paper that raised the question of whether parity was conserved in the weak interactions was an effort to understand the $\tau-\theta$ puzzle [7]. While noting that longitudinal polarization would violate parity, they did not suggest looking for it in $\beta$ decay in this first paper. Later, in a second paper proposing the two component theory of the neutrino, they specifically suggested looking for longitudinal polarization in $\beta$ decay [8]. The original experiments verifying that parity was not conserved in the weak interactions - Wu et al. [9]; Garwin et al. [10]; and Friedman and Telegdi [11], though clearly demonstrating parity violation, did not measure a longitudinal polarization.

The first measurement of longitudinal polarization in $\beta$ decay was made by Frauenfelder et al. in a very difficult measurement using Mott scattering of the electrons from ${ }^{60} \mathrm{Co} \beta$ decay. These measurements clearly showed parity violation, and gave a result for longitudinal polarization consistent with, though smaller than $\mathrm{P}=-\mathrm{v} / \mathrm{c}$ [12]. This original Mott scattering experiment was difficult for a number of reasons. Since Mott scattering only measures transverse polarization, it is necessary to electrostatically rotate the longitudinal polarization from $\beta$ decay. Such an electrostatic rotation generates very short focal length electron optics, and is generally 
astigmatic as well. Furthermore, the rotation is correct for only one energy, while $\beta$ decay gives a broad energy spectrum. Only the scattered electron is detected, making rejection of backgrounds very difficult, as there are often $\gamma$ 's associated with $\beta$ decay as well as electrons and $\gamma$ 's from beam striking the apparatus walls, rotator electrodes and beam dump. Plural and multiple scattering effects in the very thinnest of foils degrade the asymmetry, and this effect is not amenable to analytic calculation. Finally, Mott scattering does not work for positrons, removing the possibility of checking many $\beta$ decays.

These realities led Frauenfelder et al. to switch to M $\phi 1$ ler scattering for electron polarimetry, and they soon published (in the same volume of Physical Review as their earlier Mott measurements!) M $\phi 1$ ler scattering measurements of longitudinal polarization [13]. Mфller scattering had initially been observed with cloud chambers, but that technique was clearly inadequate to verify the M $\phi 1$ ler (and Bhabha, for positrons) scattering formulas. A series of counter experiments at Cornell, using a spectrometer, were reported by Ashkin et al. [14]. These experiments measured the M $\phi 1$ ler cross section about $90^{\circ}$ in the center-of-mass, and, though they did not measure an asymmetry, they clearly demonstrated that the spin dependent terms in the M $\phi$ ller formula were required to fit the experimental measurements. This paper also reported a confirmation of the Bhabha formula for positron scattering.

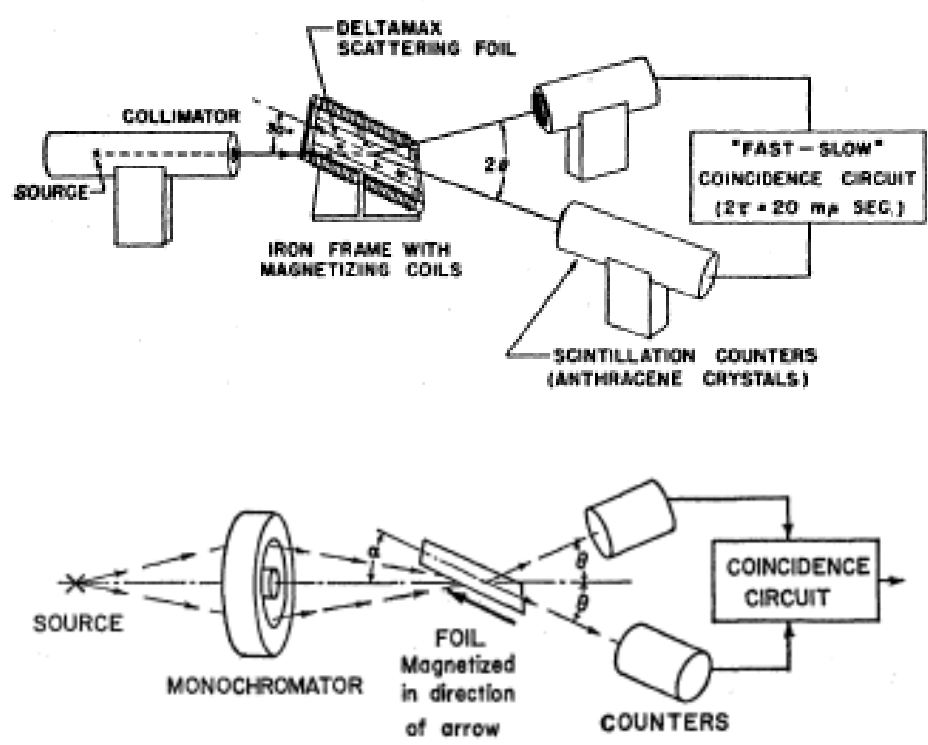

Figure 4. The first Mфller polarimeter of Frauenfelder et al., (top) and its improved version incorporating a solenoid lens as a spectrometer (bottom).

The first M $\phi$ ller scattering apparatus of Frauenfelder et al. is shown in figure 4 (top), and is simplicity itself. Coincidence detection was really the great advance with this polarimeter. The target was a foil of an iron alloy easily magnetized to saturation by a small field. The fraction of the magnetization arising from the electron spins is not known with high precision, but this was not a major problem with this experiment. Electron energies were determined from pulse height from the scintillation counters, and angles from the geometry. This first M $\phi l l e r$ measurement clearly demonstrated polarization equal to $\mathrm{v} / \mathrm{c}$ within errors, and that the electron polarization was negative. However, the technique still suffered from the presence of $\gamma$ 's. The apparatus was significantly improved by the addition of a solenoid lens with its central region blocked, which served as a momentum spectrometer, as shown in figure 4 (bottom) [ 15]. This latter paper reports a thorough discussion of systematic effects and sources of experimental uncertainty, and measured both electron and position helicities with good precision. 
Improvements on the initial Mott polarimeter were also made by other groups, and Mott scattering soon became a standard technique for the study of beta decay. Greenberg et al., made the first high quality Mott measurements [16 ]. They used a carefully designed apparatus shown in figure 5 (top), and operated at a scattering angle providing the highest efficiency, illustrated in figure 5 (bottom). This paper includes a detailed discussion of systematic effects and sources of uncertainties, and reported a value of the polarization with an uncertainty below $6 \%$.
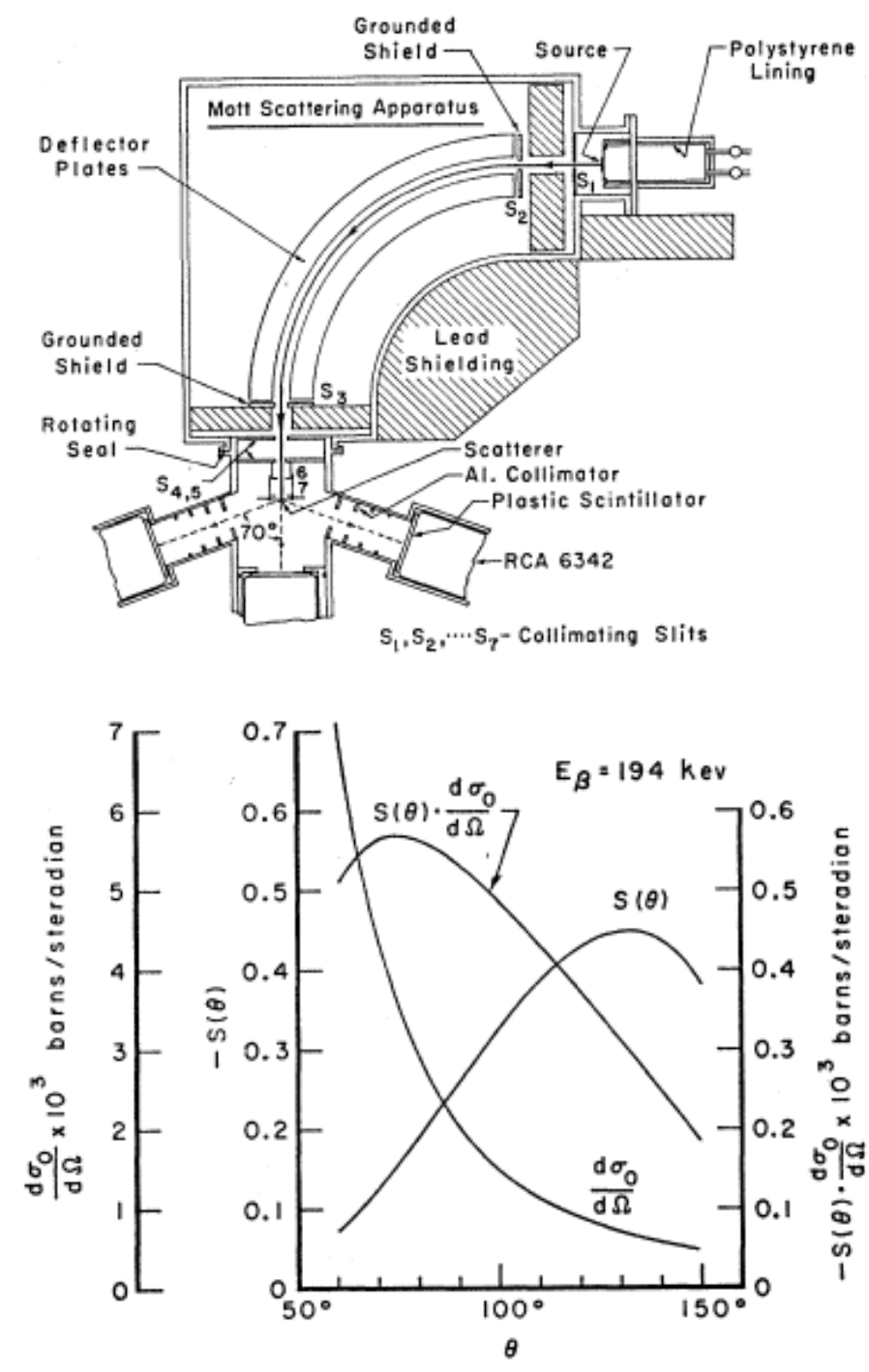

Figure 5. The Mott scattering apparatus of Greenberg et al. (top), and the selection of the optimal scattering angle (bottom).

Finally, it is worth noting that a Compton polarimeter of sorts was developed in the early parity violation days by Goldhaber et al [17]. They measured the transmission of bremsstrahlung produced by beta decay electrons through a magnetized iron block, and demonstrated that the photon circular polarization was high and consistent with being $-\mathrm{v} / \mathrm{c}$ as expected. This method was not statistically competitive with either Mott or M $\phi 1$ ler polarimeters, however. Such polarimeters have subsequently been employed at several labs (e.g. Mainz, Bates) to monitor the stability of the beam polarization during parity violation studies. 
Thus, by 1960, all three methods for the measurement of electron polarization had been demonstrated, and both Mott and Mфller scattering had been developed into reasonably well understood tools for polarization measurement in the few hundred $\mathrm{keV}$ to few $\mathrm{MeV}$ energy range. This situation was unchanged for many years, as the only source of polarized electrons was either beta decay or Mott scattering, and either provided only low to modest fluxes of low (few MeV maximum) energy electrons. The next developments in electron polarimetry would come only after the realization of polarized electron sources for accelerators, nearly 15 years later.

\section{Polarization measurements at high energies}

With the introduction of polarized electron sources for research accelerators, M $\phi$ ller polarimeters were the only polarization measurement tool available for many years. Mott scattering was suitable only at low energies, and the optical sources and accelerated electron beam intensities of the day fell far short of what was required for Compton scattering to be useful. The first polarized electron source, based on the photoionization of state-selected ${ }^{6} \mathrm{Li}$ atoms, was installed on SLAC in 1974, and the polarization of the accelerated beam measured with a single arm Moller polarimeter [18]. The "single arm" was the SLAC $8 \mathrm{GeV}$ spectrometer. This allowed excellent particle identification and separation from electrons scattered by other processes, though it was clearly not a practical choice for an experimental polarimeter.
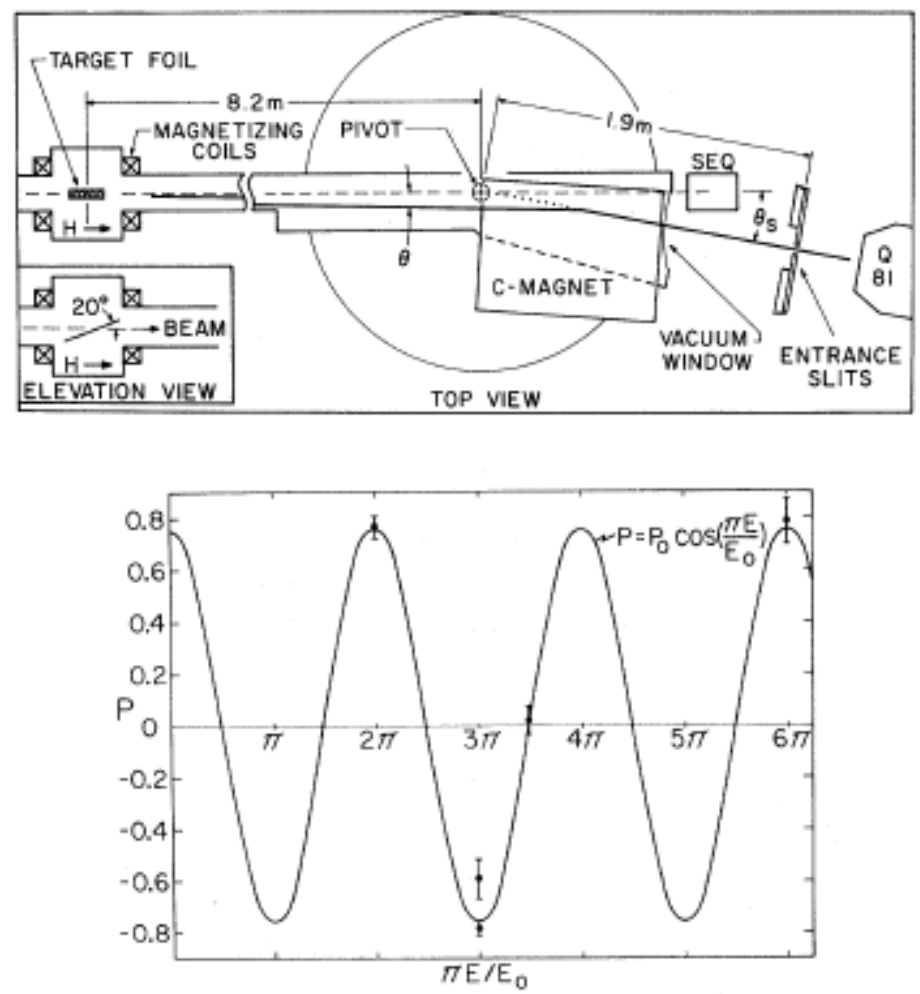

Figure 6. Plan view of the first high energy $M \phi l l e r$ scattering measurement at SLAC (top) and the measured beam polarization versus energy (bottom). Q81 is the entrance quadrupole of the $8 \mathrm{GeV}$ spectrometer. The $\mathrm{C}$ magnet had an iron shunt which allowed the primary beam to pass undeflected to the SEQ. The 
energy Eo is $3.237 \mathrm{GeV}$, which gives a total precession of $\pi$ in the $27.5^{\circ}$ deflection from the accelerator to the experiment.

The experimental setup for this measurement is shown in figure 6 (top), and the polarization measured at several beam energies in figure 6 (bottom). The beam to the experimental target was deflected by $27.5^{\circ}$ from the accelerator axis, resulting in longitudinal polarization only every $3.237 \mathrm{GeV}$. The polarized target for these measurements was a supermendur foil oriented at $20^{\circ}$ to the beam, and magnetized to saturation by a pair of air core Helmholz coils. Very similar targets were used for accelerator-based polarization measurements for many years.

In 1992 Levchuk pointed out that the target electrons are not free, but rather bound in the target atoms, leading to potentially significant systematic errors in M $\phi 1$ ler measurements [19]. The actual polarized electrons are very weakly bound, while the remaining electrons are more tightly bound, with the K-shell electrons quite tightly bound. This leads to a systematic uncertainty dependent on the detector acceptance, since the angular distribution of all unpolarized scattered electrons is broader than that of the polarized (essentially unbound) electrons. At the SLC, where both Compton and single-arm M $\phi 1 l e r$ polarimeters had been developed, Swartz et al. conducted a thorough study of the Levchuk effect for their particular M $\phi 1 l e r$ polarimeter [20]. The result was that their M $\phi$ ller polarization measurements were $14 \%$ high, compared to the Compton measurements. While the Levchuk effect may not be as prominent in double-arm coincidence M $\phi 1 l e r$ polarimeters, it must be studied or simulated in detail for every setup, and remains a significant source of systematic uncertainty in many such polarimeters.

Though M $\phi$ ller polarimeters are generally used for longitudinal polarization measurement, they also have an analyzing power $1 / 7$ as large for transverse polarization. Some groups have constructed $\mathrm{M} \phi$ ller polarimeters to measure all three polarization components. A recent example is the polarimeter constructed for the ELSA stretcher ring at Bonn [21]. This polarimeter achieved a systematic uncertainty at or below $2 \%$ for longitudinal polarization measurements.

That the beams in electron-positron storage rings should become polarized parallel (for $\mathrm{e}^{+}$) or anti-parallel (for $\mathrm{e}^{-}$) to the magnetic guide field of the ring through the emission of synchrotron radiation was predicted in the mid-1960s by Sololov and Ternov [22]. Evidence for such polarization was obtained by examining event distributions, and by Touschek (intra-beam Mфller) scattering. However, neither of these methods is suitable for on-line polarization measurements. Compton scattering offers the only practical way to study the polarization of stored beams.

By the mid-1970s, measurements at SLAC's SPEAR storage ring showed clear azimuthal asymmetries consistent with large beam polarizations in ee scattering, $\mu \mu$ production, and hadronic events [23]. The fact that the hadronic events showed a similar azimuthal distribution to the ee and $\mu \mu$ events was indicative of their origin in pairs of spin $1 / 2$ quarks, rather than the spin 0 of the pions and kaons observed. These results led to interest in constructing a Compton polarimeter for SPEAR. This was accomplished by directing the circularly polarized beam from a cavity-dumped Argon-ion laser against the positron beam of SPEAR [24]. The beam polarization was measured by the up-down asymmetry in the backscattered gammas. A Pockels cell was used to reverse the optical beam polarization at regular intervals, minimizing systematic effects. An extensive study of the positron beam polarization (without a colliding electron beam) as a function of beam energy was completed, revealing a rich structure of strong depolarizing resonances on an otherwise highly polarized beam, as shown in figure 7 (top) [25]. 
Here $v$ refers to the spin tune - the number of revolutions the spin makes per orbital revolution, and $v_{\mathrm{x}}, v_{\mathrm{y}}$, and $v_{\mathrm{s}}$ refer to the frequencies of horizontal and vertical betatron motion and synchrotron motion. The maximum possible polarization is $92.4 \%$, and is obtained in the regions between depolarizing resonances.

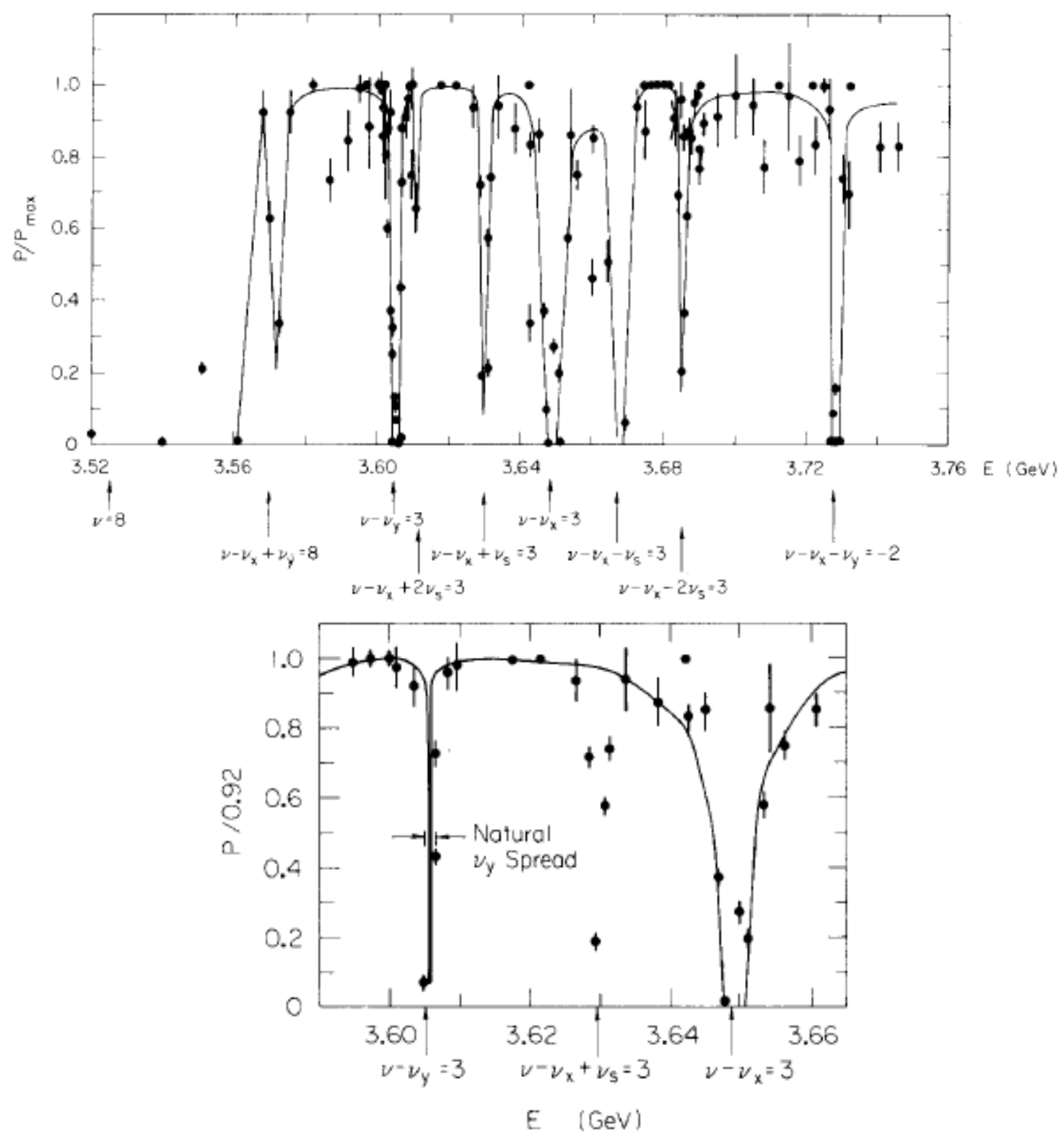

Figure 7. The rich structure of spin depolarizing resonances in the SPEAR storage ring (top), and the narrow depolarizing resonance used for the precision energy measurement (bottom).

By selecting an operating point close to a particularly narrow depolarizing resonance, as shown in figure 7 (bottom), and monitoring the polarization while carefully changing the betatron tune of the storage ring onto the resonance, it is possible to obtain a very accurate measurement of the beam energy. This technique has been employed to determine the masses of the $\Psi$ and $Y$ particles. Ultimately Compton polarimeters were developed for almost all large storage rings, and transverse polarization was observed wherever it was sought, even at $60.6 \mathrm{GeV}$ in LEP. 
However, transverse polarization was never used for other than energy determinations, and the often considered spin rotators were never used to generate longitudinal polarization in an $\mathrm{e}^{+}-\mathrm{e}^{-}$ ring.

Compton polarimeters for the measurement of longitudinal polarization were developed in the early 1990s, for measurement of the polarization at HERA, SLC, and NIKHEF [26]. In this case, rather than observing an azimuthal asymmetry, one observes a scattering (and thus energy) asymmetry in the backscattered beam. At HERA, two Compton polarimeters measured the same beam - one transversely polarized before the spin rotators, and one longitudinally polarized near the e-p interaction region. But, the bottom line is that by the late 1970s, both Moller and Compton polarimetry had been adapted for use at high beam energies, and there were few innovations after this. Once again, there was a period of relatively little development until new accelerator technologies appeared.

\section{Advances at Jefferson Laboratory}

The successful operation of the CEBAF accelerator at Jefferson Lab resulted in a significant improvement to each type of polarimeter. This was a result of the lab's delivery of beam simultaneously to three (soon to be four) experimental halls, and the $100 \%$ duty factor high average current delivered. With a little selection of the beam energies, longitudinal, or near longitudinal polarization was simultaneously available in all three halls. These advances led to a considerable growth in the number and statistical precision of polarized beam experiments, leading directly to the need for polarimeters operable at higher average current, and with improved statistical precision and thus reduced systematic uncertainties. In response to these needs, various groups developed a high energy (ca. $5 \mathrm{MeV}$ ) Mott polarimeter to measure the beam from the injector, a new M $\phi$ ller polarimeter with a normal incidence pure iron foil target, and the introduction of an optical storage cavity to allow precision Compton polarization measurements at CEBAF's average currents which, though large, are still modest by storage and stretcher ring standards.

Mott polarimetry has been typically used at energies no higher than a few hundred keV, though a series of measurements at $14 \mathrm{MeV}$ were completed at Mainz [27], following studies of time reversal invariance in the decay of ${ }^{8} \mathrm{Li}$ which used Mott scattering at these high energies [28]. There are a number of advantages to Mott scattering at these energies - for example such beams generally have RF time structure, allowing high precision non-invasive beam position and current monitoring; optical transition radiation makes the beam spot on the target foil visible, permitting study of systematic position or shape shifts with polarization reversal; time-of-flight measurements can eliminate detection of electrons scattered from other than the target foil; the small cross-sections at these energies allow use of higher average current beams; plural scattering is quite small, allowing thicker target foils; and the counting rates are large enough that use of foils of several different Z's are practical, allowing additional systematic effect studies.

Recognizing that beam depolarization in the CEBAF accelerator is negligible, and the potential advantages of higher energy Mott polarimetry, it was decided to build a precision Mott polarimeter at the nominal $5 \mathrm{MeV}$ point in the injector. The Sherman function for the energy range of interest is shown in figure 8 (top), and the results of a series of measurements for different energies and a range of $\mathrm{Au}$ foil thicknesses is shown in figure 8 (bottom). The actual polarimeter has four detectors at a mean scattering angle of $172.6^{\circ}$, each $90^{\circ}$ apart in azimuth, allowing both transverse polarization components to be measured simultaneously. Recent improvements in the time-of-flight system assure that only electrons originating from the 
scattering foil are detected, and with this advance it is planned to make another series of measurements in the near future. A detailed GEANT 4 model of the polarimeter is in development [29]. The principal systematic uncertainty of this polarimeter is knowledge of the nuclear size effect's impact on the Sherman function. With the technical improvements and the understanding gained from a detailed model, a total uncertainty below $1 \%$ is anticipated for this upgraded polarimeter.
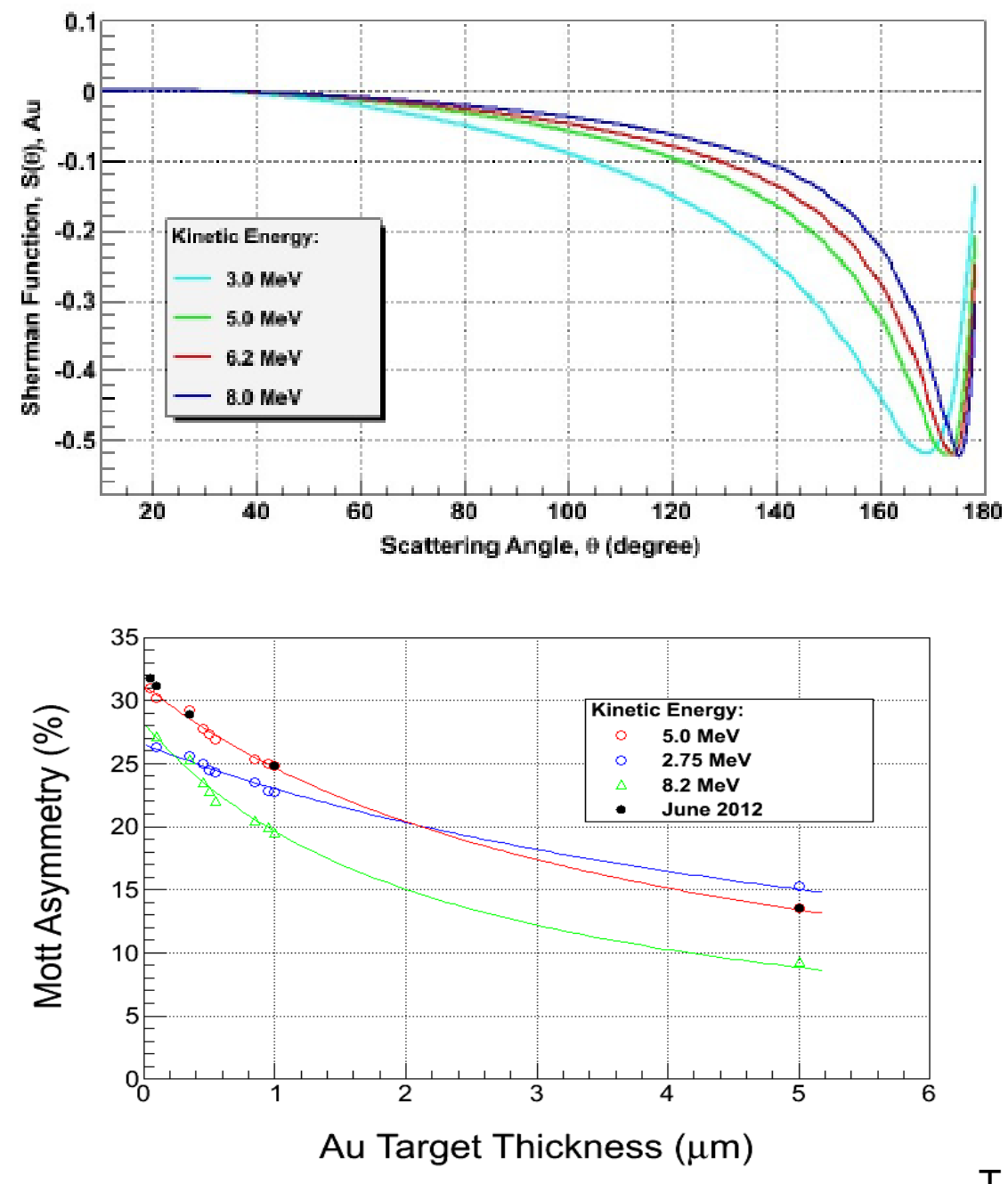

Figure 8. The calculated Sherman function for the energies of interest for the JLab Mott polarimeter (top), and measurements of the asymmetry as a function of foil thickness from 2001 (three energies) and 2012 (bottom).

A major contribution to the systematic uncertainty of all $M \phi l l e r$ polarimeters using easily magnetized (and thus alloy) target foils is knowledge of the fraction of the target magnetization coming from the alignment of the atomic electron spins. This fraction is much better known in the case of pure iron, which unfortunately is not so easily magnetized. Using a superconducting solenoid to polarize a pure iron foil perpendicular to the foil plane reduces the uncertainty in the target polarization significantly, and also allows the beam to also be perpendicular to the foil. A polarimeter of this sort was developed for use in Hall $\mathrm{C}$, with a claimed systematic uncertainty below $0.5 \%$, though this has yet to be demonstrated experimentally [30]. One difficulty with this or any other M $\phi 1$ ler polarimeter is that operation at average beam currents as high as 
routinely available at CEBAF causes very substantial target heating, reducing the polarization. Various methods such as rastering and pulsed kickers have been pursued to overcome this limitation without adding to the uncertainty budget or otherwise affecting the experiment, but no satisfactory resolution has been clearly adopted. The original developers of this polarimeter planned to use Kerr effect measurements of the target polarization at the operating temperature, and demonstrated the technique, but it has yet to be implemented. Never-the-less, this type of polarimeter target has significantly improved the systematic uncertainty budget, and similar targets will likely be used in other beamlines and labs. An acceptable solution to the target heating problem can almost certainly be engineered. Of course, even with the thinnest targets, M $\phi$ ller polarimeters are destructive to the beam, and cannot be used during experimental data taking.

In principle, Compton polarimetry should be a near ideal solution to the problem of high energy polarimetry. The polarized "target" - a laser beam - is easily manipulated and its polarization is easily controlled and measurable with very good precision. Backgrounds should be negligible, and coincidence detection is quite practical. And the target is thin enough that the polarimeter can be operated simultaneously with the experiment. However, in practice, Compton polarimetry is not so easy. A JLab group in Hall A developed an optical storage cavity to greatly increase the circulating optical power to the to the $\sim 1 \mathrm{~kW}$ level at $1.06 \mu \mathrm{m}$ wavelength. At this power level, polarization measurement at the $\sim 1 \%$ level requires some hours of operation with a $100 \mu \mathrm{A}$ beam current [31]. Such long measurement times make studies of systematic effects, polarimeter alignment, etc. very difficult. The beam setup for the Compton polarimeter has proven difficult, requiring considerable time in general. Small amounts of beam scraping or loss are troublesome, and the mirrors of the storage cavity may be damaged by synchrotron radiation and/or beam losses. The asymmetry is relatively low at low (ca. $1 \mathrm{GeV}$ ) energy. In spite of all these difficulties, the HAPPEX III parity violation experiment reported a total polarization uncertainty of $0.8 \%$ from combined Compton and M $\phi 1 l e r$ polarimetry at 3.48 $\mathrm{GeV}[32]$.

Various improvements to Compton polarimetry can be made. Perhaps the easiest of these is to shorten the laser wavelength, and $532 \mathrm{~nm}$ seems an obvious choice. Improved detectors may also help. The asymmetry will be larger with the CEBAF energy upgrade.

Overall, the high average current and $100 \%$ duty cycle of the CEBAF machine have resulted in pushing high energy polarimetry into the $1 \%$ precision range. With the coming energy upgrade, there will be considerable pressure to improve things to $0.5 \%$ or less, which will keep polarimeter builders busy for some while.

\section{Comparison of polarimeters}

With CEBAF capable of delivering highly longitudinally polarized beams simultaneously to each of three experimental halls, and with steady reductions in the claimed systematic uncertainties of the various polarimeters in use there, a comparison of the polarization measured by each polarimeter becomes attractive. Such a comparison is possible because in an accelerator like CEBAF, there is no meaningful depolarization in either the linacs or the recirculation arcs between the injector and any experimental hall, so polarization produced at the injector is transported without loss to the experimental halls. This polarization may not be oriented longitudinally, but its magnitude is not degraded.

An experiment was conducted at Jefferson Lab in 2000 to compare the polarization measured by the $5 \mathrm{MeV}$ Mott polarimeter in the injector, the Hall A M $\phi$ ller and Compton polarimeters, and 
the Hall B and Hall C M $\phi$ ller polarimeters [33]. A Wien filter in the injector allowed the polarization to be rotated through $+/-110^{\circ}$ in the injector. By making polarization measurements as a function of the polarization orientation exiting the injector, one traces out a sinusoid of polarization versus angle at each polarimeter. The amplitude of the sinusoid gives the polarization, and the phase shift with respect to the injector allows a high precision measurement of the accelerator energy. The results are shown in figure $9 \mathrm{a}$ and $9 \mathrm{~b}$.
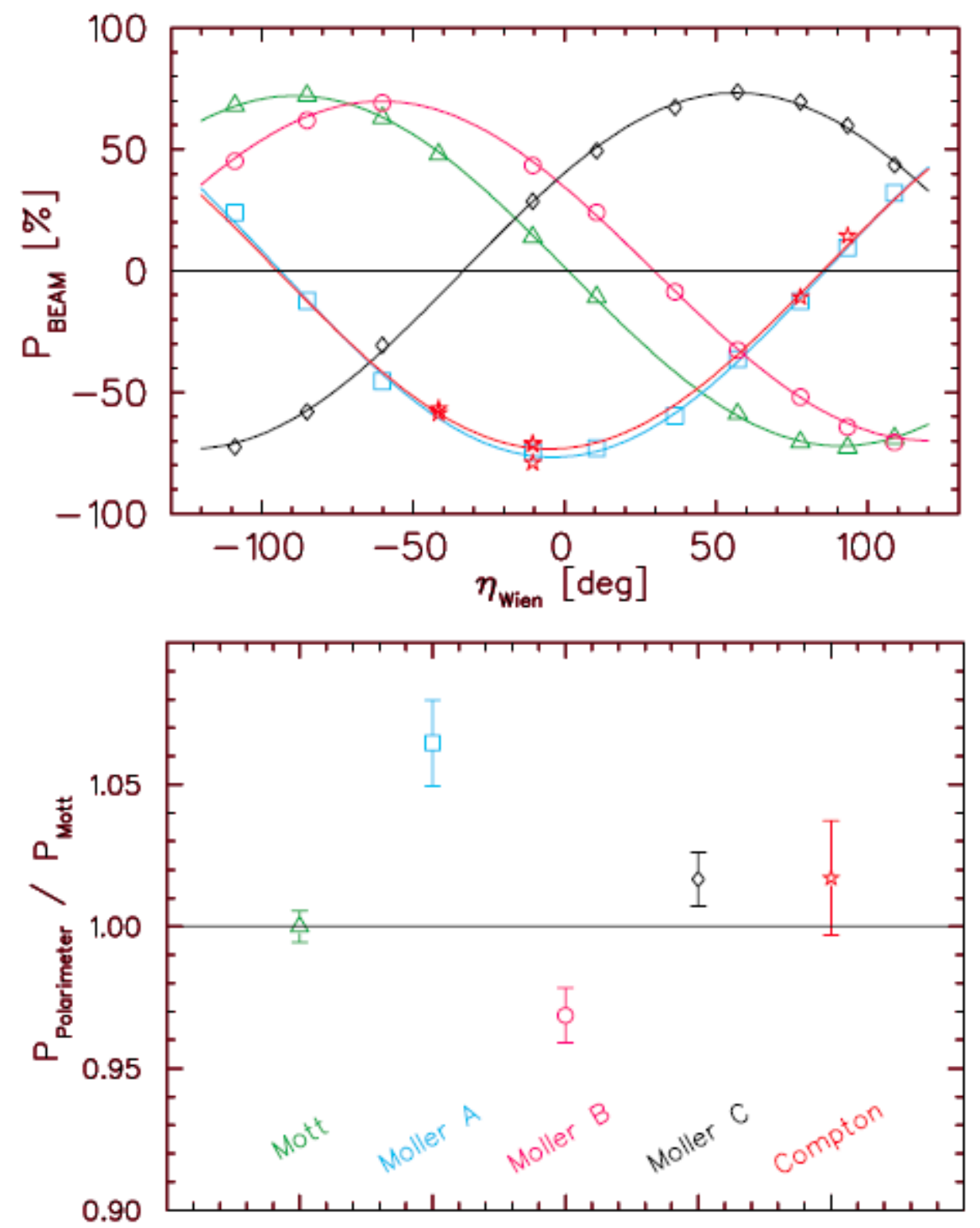

Figure 9. (a) The "spin dance" measurements made in 2000 for each of the five polarimeters, and (b) the beam polarization as reported for each polarimeter, normalized to the Mott measurements.

Clearly all these polarimeters are not in agreement, though the $5 \mathrm{MeV}$ Mott, the Hall $\mathrm{C} \mathrm{M} \phi$ ller, and the Hall A Compton are in reasonable agreement. Difficulties with the Halls A and B $\mathrm{M} \phi$ ller polarimeters were identified as a result of these measurements. Unfortunately, another polarimeter comparison done in 2006 showed significant discrepancies between these the 5 
MeV Mott and the Hall C M $\phi$ ller polarimeters. These results show that there is obviously work to be done in understanding the systematic uncertainties of these various polarimeters. Intercomparisons such as those above cannot determine which, if any, polarimeter is giving the most accurate result, but they can clearly motivate seeking a deeper understanding of all the systematic effects of each polarimeter.

\section{Summary and outlook}

Electron polarimetery was developed, and has advanced in three fairly well defined time periods, separated by 15-20 year periods of relatively little development. The first, driven by the discovery of parity violation in the weak interaction, led to the development of the first, low energy versions of all three polarimeter types - Mott, M $\phi 1 l e r$, and Compton. The second advance came with the introduction of polarized sources on the research accelerators and the development of polarization in storage rings, both delivering high energy beams. Finally, with the advent of the CEBAF accelerator with its exceptional capabilities, polarimeters were improved to approach measurement uncertainties approaching 1\%. As ever more demanding parity violation experiments are planned, there is a real need to reduce the systematic uncertainties further, with $1 / 2 \%$ an often quoted goal. Precision intercomparisons of different polarimeters, each measuring the same beam polarization, show that there is still much understanding to be gained to achieve such precision.

Brute force polarization of a dense stored volume of hydrogen atoms, with high fields and low temperatures, has been proposed as a method to make a very high polarization M $\phi$ ller target, free of many of the problems of present targets [34]. This idea looks very good on paper, but it has yet to be implemented, as the costs and effort to build such a device are large. And, no doubt building such a device would encounter difficulties not presently anticipated.

One wonders whether it might be possible to develop a $\mathrm{M} \phi$ ller target based on $2^{3} \mathrm{~S}_{1}$ metastable helium atoms, which have two identically polarized electrons. It is easy to generate large numbers of helium metastables, and they can be optically pumped to the desired state. It is also important to remember that it is not necessary to have a very highly polarized $\mathrm{M} \phi l$ ler target the current relatively low analyzing power of iron foil targets is not really an obstacle to precision polarimetry. Rather, it is important to know the analyzing power with great accuracy.

Recently there has been discussion of attempting to calibrate the JLab Mott polarimeter against an absolute standard - low energy optical polarimeters can be built for which the analyzing power can be calculated absolutely with great precision [35]. This would be a demanding effort, as shown as shown by a recent attempt at Mainz [36]. In principle it should be possible to do a comparison at the $0.4 \%$ level, possibly extending that to $0.2 \%$. Either result would represent a considerable advance in the present state-of-the-art.

Clearly electron polarimetry will be a rewarding field of effort for some time to come!

\section{References}

[1] C. Mфller, Ann. Physik 14, 531 (1932)

[2] O. Klein and Y. Nishima, Zeit. f. Phys. $\underline{\mathbf{5 2}}, 853$ (1929)

[3] N. F. Mott, Proc. Roy. Soc. (London) $\underline{\mathbf{A 1 2 4}}, 425$ (1929)

[4] C. G. Shull, C. T. Chase, and F. E. Myers, Electron Polarization, Phys. Rev. $\underline{\mathbf{6 3}}$, 29 (1943) 
[5] N. Sherman, Coulomb Scattering of Relativistic Electrons by Point Nuclei, Phys. Rev. 103, 1601 (1956)

[6] W. H. Louisell, R. W. Pidd, and H. R. Crane, An Experimental Measurement of the Gyromagnetic Ratio of the Free Electron, Phys. Rev. 94, 7 (1954)

[7] T. D. Lee and C. N. Yang, Question of Parity Conservation in Weak Interactions, Phys. Rev. 104, 254 (1956)

[8] T. D. Lee and C. N. Yang, Parity Nonconservation and a Two-Component Theory of the Neutrino, Phys. Rev. 105, 1671 (1957)

[9] C. S. Wu, E. Ambler, R. W. Hayward, D. D. Hoppes, and R. P. Hudson, Experimental Test of Parity Conservation in Beta Decay, Phys. Rev. 105, 1413 (1957)

[10] R. L. Garwin, L. M. Lederman, and M. Weinrich, Observations of the Failure of Conservation of Parity and Charge Conjugation in Meson Decays: the Magnetic Moment of the Free Muon, Phys. Rev. 105, 1415 (1957)

[11] J. I. Friedman and V. L. Telegdi, Nuclear Emulsion Evidence for Parity Nonconservation in the Decay Chain $\pi^{+}-\mu^{+}-e^{+}$, Phys. Rev. $\underline{\mathbf{1 0 5}}, 1681$ (1957)

[12] H. Frauenfelder et al., Parity and the Polarization of Electrons from $\mathrm{Co}^{60}$, Phys. Rev. 106, 386 (1957).

[13] H. Frauenfelder et al., Parity and Electron Polarization: Mфller Scattering, Phys. Rev. 106, 643 (1957).

[14] A. Ashkin, L. A. Page, and W. M. Woodward, Electron-Electron and Positron-Electron Scattering Measurements, Phys. Rev. 94, 357 (1954).

[15] J. D. Ullman et al., Determination of Electron and Positron Helicities with M申ller and Bhabha Scattering, Phys. Rev. 122, 536 (1961).

[16] J. S. Greenberg et al., Mott-Scattering Analysis of Longitudinal Polarization of Electrons from $\mathrm{Co}^{60}$, Phys. Rev. 120, 1393 (1960).

[17] M. Goldhaber, L. Grodins, and A. W. Sunyar, Evidence for Circular Polarization of Bremsstrahlung Produced by Beta Rays, Phys. Rev. 106, 826 (1957).

[18] P. S. Cooper et al., Electron-Electron Scattering at GeV Energies, Phys. Rev. Lett., $\underline{\mathbf{3 4}}, 1589$ (1975).

[19] L. G. Levchuk, Nucl. Instr. Meth. A $\underline{\mathbf{3 4 5}}, 496$ (1994).

[20] M. Swartz et al., Observation of target electron momentum effects in single-arm Mфller polarimetry, Nucl. Instr. Meth. A $\underline{\mathbf{3 6 3}}, 526$ (1995).

[21] T. Speckner et al., The GDH-M (2004).

[22] A. A. Sokolov and I. M. Ternov, Sov. Phys.-Dokl. $\underline{\mathbf{8}}, 1203$ (1964).

[23] J. G. Learned, L. G. Resvanis, and C. M. Spencer, Phys. Rev. Lett. $\underline{\mathbf{3 5}}, 1688$ (1975). R. F. Schwitters et al., Phys. Rev. Lett. $\underline{\text { 35, }} 1320$ (1975).

[24] D. B. Gustavson et al., A Backscattered Laser Poarimeter for e+e-Storage Rings, Nucl. Instr. Meth. 165, 177 (1979). 
[25] J. R. Johnson et al., Beam Polarization Measurements at the SPEAR Storage Ring, Nucl. Instr. Meth. 204, 261 (1983).

[26] D. Calloway et al., SLAC-PUB 6423m 1994; W Lorenzon, AIP Conf. Proc. 521, AIP, New York, 1998, p. 181; I. Passchier et al., A Compton backscattering polarimeter for measurement of longitudinal electron polarization, Nucl Instr. Meth. in Phys. Res. A 414, 446 (1998).

[27] J. Sromicki et al., Polarization in Mott Scattering of Multi-MeV Electrons from Heavy Nuclei, Phys. Rev. Lett. 82, 57 (1999).

[28] J. Sromicki et al., Study of time reversal violation in $\beta$ decay of polarized ${ }^{8}$ Li, Phys. Rev. C $\underline{\mathbf{5 3}}$, 932 (1996).

[29] M. McHugh et al, contribution to this workshop.

[30] M. Hauger et al., Nucl. Instr. Meth. A 462, 382 (2001).

[31] M. Baylac et al., First electron beam polarization measurements with a Compton polarimeter at Jefferson Laboratory, Phys. Lett. B 539, 8 (2002).

[32] Z. Ahmed et al., New Precision Limit on the Strange Vector Form Factors of the Proton, Phys. Rev. Lett. 108, 102001 (2012).

[33] J. Grames et al., Unique electron polarimeter analyzing power comparison and precision spinbased energy measurement, Phys. Rev. ST-AB ㅍ, 042802 (2004).

[34] E. Chudakov and V. Luppov, M pller Polarimetry with Atomic Hydrogen Targets, IEEE Trans. Nucl. Sci. 51, 1533 (2004).

[35] T. J. Gay et al., Optical electron polarimetry with heavy noble gases, Phys. Rev. A $\underline{\mathbf{5 3}}, 1623$ (1996).

[36] B. Collin et al., First attempt of the measurement of the beam polarization at an accelerator with the optical electron polarimeter POLO, Nucl. Instr. Meth in Phys. Res. A 534, 361 (2004). 